\title{
Off-Label-Use bei Seltenen Erkrankungen (SE): Myasthenia gravis, Lambert-Eaton myasthenisches Syndrom und kongenitale myasthenische Syndrome
}

\section{Off-Label Use in Rare Diseases: Myasthenia gravis, Lambert-Eaton Myasthenic Syndrome and Congenital Myasthenic Syndromes}

\author{
Autor \\ Rudolf W. C. Janzen \\ Institut \\ Ehem. Chefarzt, Neurologische Klinik am Krankenhaus \\ Nordwest, Franfurt Main \\ Schlüsselwörter \\ Off-Label-Use, Lambert-Eaton-Myasthenisches-Syndrom, \\ kongenitale myasthenische Syndrome, therapierefraktäre \\ Myasthenia gravis \\ Keywords \\ off-label use, Lambert-Eaton myasthenic syndrome, \\ congenital myasthenic syndrome, refractory myasthenia \\ gravis \\ Bibliografie \\ DOI https://doi.org/10.1055/s-0044-100360 \\ Akt Neurol 2018; 45: 305-311 \\ (c) Georg Thieme Verlag KG Stuttgart · New York \\ ISSN 0302-4350
}

Korrespondenzadresse

Prof. Dr. med. Rudolf W. C. Janzen, Landwehrweg 12 C, 61350 Bad Homburg info@rwcjanzen.de

\section{ZUSAMMENFASSUNG}

Der Einsatz von Substanzen im Off-Label-Use ist weit verbreitet und mit einem erhöhten Risiko von unerwünschten Arzneimittelwirkungen (UAW) verbunden, zumal wenn die zugrunde liegende Evidenz gering ist. Dies gilt auch für die Behandlung seltener <5/10000) Erkrankungen mit ver- schiedenen myasthenen Syndromen. Die fachlichen Vorgaben für die Indikation eines Off-Label-Use sind in den spezifischen Leitlinien maßgeblich dargelegt, dabei sind die Regulationen des deutschen Gesundheitssystems zu beachten. Basis einer medikamentösen Langzeittherapie ist eine optimierte Standardtherapie, die auf dem Einsatz von Substanzen beruht, die in die Arzneimittel-Richtlinien aufgenommen sind. Die aktuellen Kriterien für die Indikation und die Überwachung eines Off-Label-Einsatzes werden dargestellt. Die Bewertung von besonderen Konstellationen, insbesondere die Therapieresistenz wird analysiert und praktische Hinweise für eine regulatorische Handhabung aufgezeigt. Derzeit führt eine Eskalation der medikamentösen Therapie immer zu einem Off-Label-Use, der als individueller Heilversuch der Begründung und eines persönlichen kontinuierlichen Monitorings bedarf.

\section{ABSTRACT}

The off-label use of licensed drugs is widespread and the risk of adverse events is high, especially if the scientific evidence in support of its use is low. The treatment of rare diseases $(<5 / 10,000)$ therefore may increase the risk of an offlabel use. The optimized standard treatment is based on the regulatory decisions of the Federal Joint Committee. The valid guidelines and the regulations of the German health system are discussed. The criteria for indication and monitoring off-label use are shown, especially focusing on the problem of refractory myasthenia gravis. Escalation of treatment results regularly in an off-label use that needs to be justified and monitored continuously. Some new aspects of the European regulations are discussed.

\section{Hintergrund}

\section{Off-Label-Use}

Ein Off-Label-Use liegt dann vor, wenn die Anwendung einer zugelassenen Substanz außerhalb des bestimmungsgemäßen Gebrauchs erfolgt. Der bestimmungsgemäße Gebrauch ist in der amtlichen Fachinformation dokumentiert und basiert auf der zugehörigen Monografie des BfArM [1]. Nach einer EU-Studie aus 2017 liegt die generelle Verschreibung einer Substanz im Off-Label-Use bei Erwachsenen zwischen 7-95\% im stationären Bereich, in der Ambulanz zwischen 6-75\% [2]. In zahlreichen Leitlinien der AWMF werden Substanzen im Off-Label-Use mit ihrem Evidenzniveau und einer Bewertung dargelegt. Ein Off-Label-Einsatz kann dann für den Arzt geboten sein, wenn 
diese Leitlinien die Bedingungen für die medikamentöse Behandlung klar formuliert haben und der Off-Label-Use den Anforderungen der verschiedenen Urteile des Bundessozialgerichts bzw. einschlägiger Urteile des Bundesverfassungsgerichts erfüllt [3-6] oder für die Erkrankung keine On-LabelTherapie vorhanden ist [7]. Eine Kostenübernahme durch die GKV kann grundsätzlich nur erfolgen, wenn vor Therapiebeginn ein begründender Antrag durch den behandelnden Neurologen gestellt wird sowie ein positives Votum des MDK und/oder eine Zustimmung der GKV vorhanden sind. Das Ausmaß des so antragsgemäß zustande gekommenen Off-Label-Use bzw. der außerhalb dieses Verfahrens stattfindende Off-Label-Use („tolerierter" Off-Label-Use) sind in Deutschland nicht systematisch erforscht. Bezüglich einer Haftung ist darauf hinzuweisen, dass der Hersteller, der von einem verbreiteten Off-Label-Use eines Produkts Kenntnis hat, gemäß §84 AMG in der Haftung gesehen werden muss [3].

Ein Off-Label-Use ist grundsätzlich mit einem erhöhten Risiko von unerwünschten Arzneimittelwirkungen (UAW) verbunden, wie die kürzlich vorgelegten Ergebnisse einer kanadischen Kohortenstudie zeigen [7]. Danach lag die durchschnittliche Rate der UAWs bei allgemeinmedizinischen Patienten unter On-Label-Use bei 12,5/10000 Patientenmonaten gegenüber $19,1 / 10000$ Patientenmonaten in einer Patientengruppe mit Off-Label-Use [7]. Solche Ergebnisse waren zusammen mit der aktuellen EU-Studie [2] die Grundlage für die Initiative „Good Off-Label Use Practices“ des Braincouncil [8]. Kern ist die Forderung einer deutlich verbesserten Pharmakovigilanz. Dies wird von der European Myasthenia Gravis Association (EuMGA) unterstützt.

\section{Seltene Erkrankungen: Beispiel Myasthenia gravis}

Seit 2000 sind in der EU Erkrankungen mit einer Prävalenz von < 5 von 10000 Personen als „rare disease“ (Seltene Erkrankung (SE)) definiert. Dies trifft nach Aussage von Orphanet auf die Myasthenia gravis (MG) in Europa zu [9] , Prävalenz: 1-9/100000. Die epidemiologische Untersuchung von Boldingh et al. [10] ergab für die AChR-positive MG eine Prävalenz zwischen 11,1 bis 14,3/100000. In Kanada zeigte sich eine geringe Zunahme der Prävalenz von 16,3/100 000 (1996) zu 26,3/100000 (2013), die z.T. auf die verbesserten Therapiemöglichkeiten und Diagnostik bezogen wird [11]. Dagegen scheinen jährliche Neuerkrankungen an MG, dort zwischen 2,3 und 2,7/100000 angegeben, eher stabil zu sein [11].

\section{Zugelassene Substanzen und vom G-BA in die Arzneimittelrichtlinien (AM-RL) aufgenommene Substanzen}

In Deutschland sind durch das BfArM für das „Anwendungsgebiet“ Myasthenia gravis einige Substanzen zugelassen ( $\triangleright$ Abb. 1). Es handelt sich meist um Altsubstanzen. Diese wurden aber nur zum Teil in den Aufbereitungskommissionen (hier: Aufbereitungskommission B3 des Bundesgesundheitsamtes) behandelt. Deren Arbeit wurde im Jahre 1986 eingestellt [12]. Azathioprin wurde 2004 im Nachzulassungsverfahren für das Anwendungsgebiet „Myasthenia gravis“ zugelassen. Für Ambenoniumchlorid, das bis Ende 1991 noch fiktiv in Deutschland zugelassen war, wurde kein Nachzulassungsantrag gestellt. Eine Anwendung von Ambenoniumchlorid ist zwar in Deutschland möglich, allerdings ist ein Import (gemäß AMG §73 Abs.3) aus einem der EU-Mitgliedsländer (Frankreich, Polen, Ungarn) erforderlich, der Gebrauch wie ein Off-Label-Use zu behandeln.

Seit Einführung der Expertengruppen Off-Label 2006 am BfArM konnte für einige Substanzen gemäß §35 c SGB V eine Regelung durch Beschlüsse des G-BA gefunden werden [13]: Mycophenolat Mofetil (MMF; 3.12.2013) und intravenöses Immunglobulin G (IVIG; 10.9.2013). Diese dürfen im Off-LabelUse gemäß AM-RL Anlage VI Teil A ohne vorlaufenden Antrag durch den behandelnden Arzt eingesetzt werden. So wurde die Basistherapie erweitert, sodass sie als „optimierte Standardtherapie“ zu bezeichnen wäre (vgl. \Abb.1). Die Kosten werden von der GKV übernommen und führen nicht zu einem Regress [4].

\section{Anwendungspraxis}

Die in Deutschland für das Anwendungsgebiet Myasthenia gravis zugelassenen Substanzen werden in den aktuellen Leitlinien der DGN (AWMF-Registernummer: 030/087) als Basistherapie behandelt [14]. Nicht alle zugelassenen Substanzen werden auch tatsächlich zur Behandlung der Myasthenia gravis eingesetzt.

Eine Untersuchung in Norwegen [15] analysierte das gesamte Spektrum verordneter Substanzen bei 850 MG-Patienten. Von 527 MG-Patienten unter Immunsuppressiva (IS) betrug das Verhältnis Azathioprin vs. Steroid 32:60\%, in einer Umfrage in Deutschland $77: 14$ \% [16; s. D Tab. 1 ). Für die Langzeittherapie ergab sich in einer Umfrage der Deutschen Myasthenie Gesellschaft (DMG) ein Verteilungsverhältnis der Substanzgruppen wie in $\mathbf{A} \mathbf{A b} \mathbf{b} \mathbf{2}$ dargestellt.

Der Nachweis neuer Autoantikörper bei MG hat neue Varianten identifiziert (z.B. MuSK-pos-MG [17] ), die eine deutlich differentere Dosierung und Therapieplanung erfordern als die AChR-AK-positive Myasthenie [18, 19].

- Tab. 1 Einsatz von Kortikoiden und Immunsuppressiva zur Behandlung von Myasthenia gravis. Norwegen in der Periode 2004 - 2010: Von 830 MG Patienten unter Pyridostigmin verwenden 636 ein Immunsuppressivum (Daten aus [15]). In Deutschland nahmen in der DMG-Umfrage 2007 von 899 befragten MG-Patienten 744 ein Immunsuppressivum (unveröffentlichte Daten).

\begin{tabular}{|l|c|c|c|c|}
\hline & \multicolumn{2}{|c|}{$\begin{array}{l}\text { Norwegen } \\
\mathbf{2 0 0 4 - 2 0 1 0}\end{array}$} & \multicolumn{2}{c|}{ Deutschland 2007 } \\
\hline Immunsuppressivum & $\mathbf{N}=\mathbf{6 3 6}$ & $\%$ & $\mathbf{N}=\mathbf{7 4 4}$ & $\%$ \\
\hline Azathioprin & 201 & 32 & 570 & 77 \\
\hline Kortikosteroid & 387 & 61 & 107 & 14 \\
\hline Mycophenolat Mofetil & 23 & 4 & 42 & 6 \\
\hline Ciclosporin & 15 & 2 & 15 & 2 \\
\hline Methotrexat & 10 & 2 & 8 & 1 \\
\hline Cyclophposphamid & 0 & 0 & 2 & $<1$ \\
\hline
\end{tabular}




\begin{tabular}{|c|c|c|c|c|}
\hline Off-Label & On-Label & Orphan drug & LL & Maßnahme \\
\hline & & & & Thym(om)ektomie \\
\hline & & & & Steroide \\
\hline & & & & Azathioprin \\
\hline SozG & & & & Methotrexat \\
\hline AM-RL + & & & & Mycophenolat Mofetil ${ }^{* * *}$ \\
\hline SozG & Japan & & & Tacrolimus \\
\hline SozG & & & & Cyclosporin A \\
\hline SozG & & & & Cyclophosphamid \\
\hline AM-RL + & & & & high-dose IVlg*** \\
\hline \multirow[t]{2}{*}{ SozG } & & & & Intervallgabe IVlg \\
\hline & & & & Immunadsorption IAD \\
\hline \multirow[t]{2}{*}{ SozG } & & & & intermittierende IAD \\
\hline & & & & Plasmaaustausch \\
\hline \multirow[t]{2}{*}{ SozG } & & & & Rituximab \\
\hline & & EMA & & Eculizumab \\
\hline \multirow[t]{5}{*}{ SozG } & & & & andere monoklonale AK \\
\hline & & & & Antisense-Therapie \\
\hline & & & & Etanercept \\
\hline & & & & therapeutische Vaccination \\
\hline & & & & Pyridostigminbromid \\
\hline \multirow[t]{3}{*}{ SozG } & Frankreich & & & Ambenoniumchlorid* \\
\hline & & & & Edrophoniumchlorid** \\
\hline & NRF Nr. 22.3 & EMA & & 3,4-Diaminopyridin (DAP) \\
\hline
\end{tabular}

- Abb. 1 Überblick und Zulassungsstatus der Behandlungsverfahren bei Myasthenia gravis (Pharmakotherapie, Interventionen und Operationen) in Anlehnung an die Leitlinie (LL) der DGN (graue Spalte). Orphan-Drug-Status und On-Label-Use sind mit Angabe der Region gekennzeichnet (grün). Substanzen im On-Label-Use und im Off-Label-Use als zugelassene Substanz nach AM-RL (AM-RL+) sind fett gedruckt. Der sonstige Off-Label-Use erfordert den Antragsweg, evtl. Klageweg (SozG) bei Nicht-Zustimmung der GKV. * ausschließlich für Myasthenia gravis zugelassen in Frankreich, Polen, Ungarn; ${ }^{* *}$ nur in Spanien für einen pharmakologischen Test zugelassen; ${ }^{* * *}$ durch Beschluss des G-BA in die AM-RL Anlage VI Teil A aufgenommen, erstattungsfähig und verordnungsfähig im Off-Label-Use ohne Antrag. 


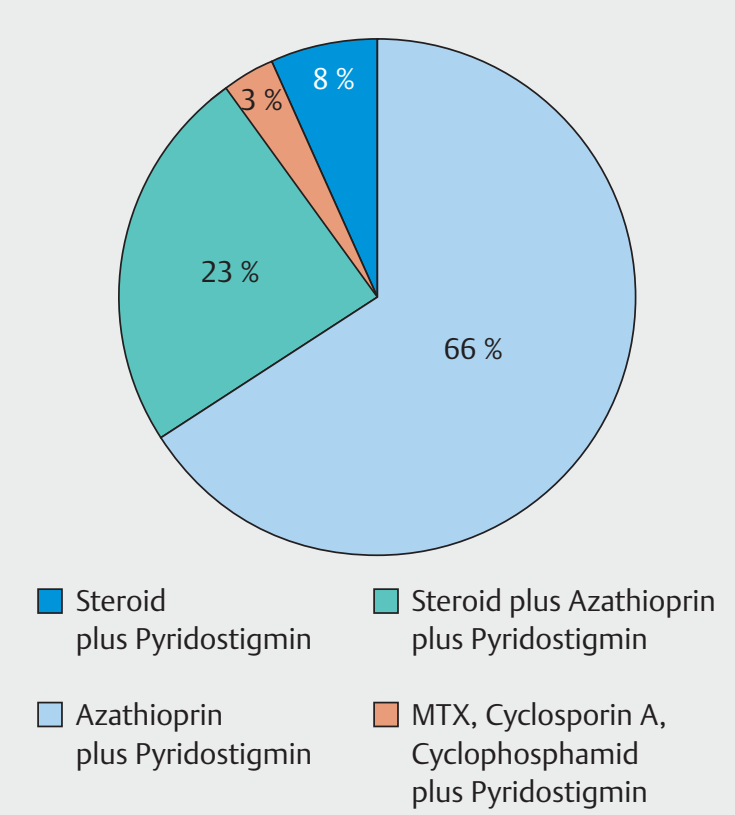

Abb. 2 Spektrum und Anteil der eingesetzten Medikamente in der Langzeittherapie bei 763 Myasthenie-Patienten. Deutsche Myasthenie Gesellschaft (DMG), modifiziert nach Daten aus Wöhrle [16].

- Tab.2 Empfehlungen für die Indikation und das Monitoring eines geplanten Heilversuchs [3].

1. umfassende Dokumentation Therapieresistenz

2. umfassende Aufklärung

3. Sicherungsaufklärung

4. Selbstbestimmungsaufklärung

5. schriftliche Einverständniserklärung

6. umfassende Dokumentation des Verlaufs (exakt, engmaschig, persönlich)

\section{Regulationskonzept zum Off-Label-Use Rare disease (orphan) designation}

Die Voraussetzungen für eine Verbesserung der Evidenz der Zulassung neuer Substanzen für die SE waren bislang aufwendig, für einen pharmazeutischen Hersteller vermutlich wenig attraktiv, um klassische RCT-Studien, z.B. zur Erweiterung der bisherigen Zulassung, aufzunehmen. Das IQWiG hat in seinem "Rapid Report" darauf hingewiesen, dass das methodische Vorgehen bei SE wie bei Nicht-SE nahezu gleich gesetzt werden kann [3]. Allerdings gibt es auch innerhalb der SE die „singulären Fälle“ [10] oder „Einzelfälle“, die auch als „ultra rare diseases" (Prävalenz: < 1:10000) zusammengefasst werden. Hierunter fällt z. B. die MuSK-pos-MG (Prävalenz 0,05-0,65/100 000 [10] oder CMS [20]. Daher haben Vrinten et al. [21] für solche ultra-rare-diseases das Studienkonzept n-of-1-Trial (sog. single case trial) am Beispiel Ephedrin als Add-on-Therapie bei MG in die Diskussion eingebracht. Arzneimittelrechtlich wurde für SE und ihre seltenen Konstellationen das Konzept der „rare disease (orphan) designation " (früher: orphan drug designation) durch der EMA eingeführt. Dies hat z. B. bewirkt, dass die seit über 40 Jahren bekannte und angewandte Substanz 3,4-Diaminopyridin, die nach NRF (Neues Rezept Formularium; Nr. 22.3) in Deutschland vom Apotheker zubereitet werden kann und auch zur Behandlung des LEMS als leitliniengerechte Therapie gilt, von der EMA nach dem erfolgreichen Zulassungsverfahren als orphan drug (Amifampridin) zugelassen wurde. Die erhöhten Kosten durch dieses Verfahren, aber auch das Verfahren selbst, wurden sehr kritisch diskutiert [22].

Von den derzeit in klinischen Studien untersuchten monoklonalen Antikörpern hat Eculizumab eine rare disease (orphan) designation für Myasthenia gravis erhalten (EMA: 29.7.2014), d. h. der EMA lagen vonseiten des Herstellers Belege für einen "significant benefit“ (Abs. 3 der EU-Richtlinie Nr. 141/2000) vor [23,24]. Inzwischen ist Eculizumab für die Behandlung einer „therapierefraktären generalisierten MG mit nachgewiesenen Antikörpern gegen Acetylcholin-Rezeptor-Protein“ zugelassen (14.8.2017) [25]. Nach Zulassung als orphan drug durch die EMA ist in Deutschland das AMNOG-Verfahren zur Bewertung eines Zusatznutzens nur dann erforderlich, wenn der Umsatz eine festgelegte Summe (derzeit 50 Mio Euro) überschreitet. Eculizumab unterliegt als Altsubstanz keinem AMNOG-Verfahren.

\section{Aufgabe der Expertengruppe (Neurologie/Psychiatrie) am BfArM}

In Deutschland wird derzeit zur Regulierung des Off-Label-Use gemäß SGB V der Ansatz verfolgt, dass die 2006 gegründeten Expertengruppen Off-Label (Neurologie/Psychiatrie) am BfArM mit einer Bewertung von Substanzen vom G-BA beauftragt wird. Diese Bewertung setzt einen Antrag der GKV, KBV oder Selbsthilfegruppen beim G-BA voraus, der die Voraussetzungen für das Verfahren prüft. Die Bewertung wird durch einen von der zuständigen Expertengruppe benannten externen Experten vorbereitet. Die dann erfolgende Bewertung durch die Expertengruppe wird nach einem abgestimmten Methoden-Papier erstellt und als konsentierter Bewertungsvorschlag dem G-BA übermittelt, der dann nach einem Stellungnahmeverfahren einen Beschluss fasst. Entweder wird die Substanz damit im Teil A der AM-RL VI (positiv) oder im Teil B (keine Off-Label-Anwendung möglich) aufgenommen. Substanzen im Teil A können ohne vorherige Anfrage bei der GKV direkt verordnet und zu Lasten der GKV eingesetzt werden, wenn die im Beschluss des G-BA genannten Vorgaben eingehalten werden, die quasi eine Erweiterung der Fachinformation sind. Die so verordnungsfähige und von der GKV übernommene Behandlung mit MMF und IVIG ist ein positives Ergebnis diese Verfahrens, erfordert aber eine besondere Aufklärung und Monitoring $([3,4]$; - Tab.2).

Verlangt der klinische Verlauf im Einzelfall eine Erweiterung der Behandlung mit Substanzen im Off-Label-Use, wie sie z.B. mit Second-Line-Substanzen nach der aktuellen Leitlinie angezeigt ist, die aber bislang nicht von der Expertengruppe bewertet wurden, muss ein vorlaufender, qualifiziert begründeter Antrag bei der zuständigen Krankenkasse gestellt werden. In dem 
- Tab. 3 Kriterien für eine therapierefraktäre Myasthenia gravis [30,31].

1. schwere, generalisierte MG mit unzureichendem Ansprechen auf eine adäquate Dosierung und Dauer einer Immunsuppression

2. häufige myasthene Krisen und/oder schwere Exazerbationen

3. wiederholte Interventionen (IVIG oder IAD oder Plasmaaustausch)

4. Gefahr der intermittierenden Heimbeatmung

5. gravierende UAWs unter der optimierten Standardtherapie

6. erhöhte individuelle Risiken (z. B. Komorbiditäten)

7. Kontraindikationen gegen die leitliniengerechte Behandlung

Antrag muss das wissenschaftliche Erkenntnismaterial, z.B. nach Stand der Leitlinie, und der Stand der klinischen Expertise im Hinblick auf eine fallindividuelle Analyse aufgearbeitet werden $[1,13,14]$. Der Antrag wird in der Regel vom MDK geprüft und dann, wenn die Kriterien des BSG-Urteils vom 19.3.2002 (sog. Sandoglobin-Urteil) bzw. die Kriterien des sog. NikolausUrteils (6.12.2006) erfüllt sind [4-6], meist genehmigt. Leider wird nicht immer die Erfahrung des behandelnden Arztes und/ oder Myasthenie-Experten ausreichend berücksichtigt. Ein ablehnender Bescheid kann bedeuten, dass - in dringlichen Fällen - sogar der Weg der einstweiligen Verfügung beschritten werden muss.

Langfristig ist nach den EU-Richtlinien die Einrichtung von krankheitsspezifischen „Centers of Expertise“ (CoE) für solche Aufgaben vorgesehen, wie sie in Deutschland von der „Nationalen Allianz für Menschen mit Seltenen Erkrankungen“ (NAMSE) auf den Weg gebracht wurde. Derzeit sollten die zertifizierten integrierten Myasthenie-Zentren (iMZ; [26]) oder spezialisierten Myasthenie-Ambulanzen angefragt werden.

\section{Myasthenia gravis \\ Optimierte Standardtherapie}

Die optimierte Standardtherapie ist eine erweiterte Basistherapie, die neben einer symptomatischen, bedarfsweisen Gabe von Pyridostigminbromid [27] in der Regel eine antiinflammatorische Behandlung mit Steroiden (Therapieziel: Induktion einer Remission) umfasst, dann eine additive Langzeitimmunsuppression mit Azathioprin, die steroidsparend wirkt und die Aufrechterhaltung und Besserung der erreichten Remission (Therapieziel: Remissionserhaltung) bewirken soll. Bei einer Azathioprin-Unverträglichkeit oder Unwirksamkeit bei angemessener Dosierung und Anwendungsdauer (oder auch bei nicht möglicher Reduktion von Steroiden unter die Cushing-Schwelle) kann Azathioprin durch Mycophenolat Mophetil ersetzt werden. Bei einer myasthenen Exazerbation kann IVIG, auch ambulant, als Add-on-Therapie zur Stabilisierung eingesetzt werden $[28,29]$.

\section{Non-Responder}

Etwa $14.8 \%$ aller Myasthenia gravis-Patienten können von einer optimierten Standardtherapie nicht hinreichend profitieren [14, 30, 31]. Diese MG-Patienten sind z. B. oft Steroidresponder, d.h. unter einer Steroiddosis, die allerdings meist deutlich über der Cushing-Schwelle liegt, sind sie gebessert und stabil, oder sie benötigen Interventionen mit IVIG oder Plasmaaustausch. Dennoch sind sie als Non-Responder unter optimierter Standardtherapie zu betrachten, da die zu befürchtenden UAWs einer meist erforderlichen Langzeittherapie mit Steroiden nicht akzeptiert werden dürfen [14, 28,32-34]. In diesen Fällen wird eine immunsuppressive Therapie im Off-Label-Use eingesetzt (second line; z.B. Methotrexat, Ciclosporin A, Tacrolimus), d.h. (s. Abb.1). Diese Therapie entspricht einem individuellen Heilversuch, der eine umfassende Aufklärung und Dokumentation erfordert ( Tab. 2; $[3,4,14]$ ). Die Situation in der Therapie bei juveniler MG ist eher noch komplexer [35].

\section{Therapierefraktäre Myasthenia gravis}

Es gibt wenige Patienten, wahrscheinlich deutlich unter 15\% mit generalisierter MG, die sich durch eine erweiterte optimierte Standardtherapie, d.h. auch nach Einsatz von Second-LineImmunsuppressiva (z. B. Methotrexat oder Ciclosporin A) nicht ausreichend bessern. Diese Konstellation im Verlauf einer generalisierten, oft fluktuierenden generalisierten MG wird als therapierefraktäre MG bezeichnet werden. Die Kriterien einer therapierefraktären generalisierten MG (TRgMG) sind im Einzelnen nicht konsentiert (vgl. > Tab. 3 [30, 31, 34, 39]). Daher sollte bei Prüfung der Evidenz und des Nutzen-Risiko-Profils bei einem beabsichtigten Therapieschritt der Einzelfall, d.h. „singulärer Fall“ (z.B. MuSK-positive Myasthenia gravis) zugrunde gelegt werden. Dies ist bei der MG schon wegen der sehr unterschiedlichen individuellen Verteilung der Muskelschwäche und Krisenanfälligkeit sinnvoll. Eine individuelle Nutzen-Risiko-Bewertung ist dabei, nicht zuletzt für die partizipative Patientenentscheidung ein zusätzliches sehr wesentliches Entscheidungskriterium $[14,19,28,32]$.

Eine erweiterte Eskalationstherapie kann dann bedeuten, dass zusätzlich zur immunsuppressiven Therapie entweder die Indikation für eine stabilisierende Langzeittherapie mittels intermittierender IVIG-Gabe oder - selten - intermittierender Langzeit-IAD [36] bzw. Langzeit-Plasmaaustausch als sog. Addon-Therapie zu erwägen ist. Auch der Versuch einer Remissionsinduktion mittels Einsatz von monoklonalen Antikörpern, z. B. Rituximab im Off-Label-Use, hat sich bewährt [14, 17, 19,37 -39]. Die Anwendung von Rituximab, insbesondere bei MuSKpositiver Myasthenia gravis, ist seit über 10 Jahren eine favorisierte, erfolgreiche Strategie [39]. Die Wirksamkeit und die Nutzen-Risiko-Bewertung werden überwiegend positiv gesehen. Von den in den Leitlinien genannten weiteren monoklonalen Antikörpern ist Eculizumab von der EMA zugelassen und fungiert als Add-on-Therapie zur laufenden Behandlung bei TRgMG mit nachgewiesenen AChR-Antikörpern. Als Altsubstanz unterliegt Eculizumab nicht dem AMNOG-Verfahren. Die differenzierte Indikationsfindung innerhalb der deutschen Behandlungspraxis, die auf einer Expertise von mit deutlich über 10 Jahren fußt („well established use“), hat gerade begonnen. Daher sind viele Fragen noch unbeantwortbar, die Anwendung wird sich auf besondere Fälle mit generalisierter MG begrenzen.

Es gibt sehr seltene Konstellationen von definitiv nicht ausreichend behandelbarer MG, deren Entstehung nicht immer verstanden wird (sog. ausgebrannte Myasthenie, areaktive MG). Ob diese Verläufe mit den modernen Therapiemöglichkei- 
ten zukünftig vermieden werden können, wird sich erweisen. Die schwerste Beeinträchtigung ist die Atemlähmung. Die Indikation für eine Heimbeatmung in diesen Fällen muss in der Zusammenarbeit von Neurologen und Pulmologen erarbeitet werden [41].

\section{Lambert-Eaton-Myasthenie-Syndrom (LEMS)}

Die immunsuppressive Behandlung des idiopathischen und paraneoplastischen LEMS (Prävalenz: 0,1-0,9/100000 [9]) mit Steroiden und/oder Azathioprin wird analog wie bei MG-Patienten angewandt [29, 42], wenngleich nur im Rahmen des Off-Label-Use, da eine explizite Zulassung für dieses Anwendungsgebiet aufgrund fehlender Studiendaten nicht vorliegt. Diese Therapie ist aber leitliniengerecht. Die symptomatische Therapie erfolgt mit 3,4-Diaminopyridin (Amifampridin). Dieses steht sowohl als Rezeptur nach Neues Rezept Formularium [NRF 22.3. $5 \mathrm{mg}$ bzw. $10 \mathrm{mg}$ ] als auch als Originalpräparat zur Verfügung. Die Zulassung erfolgte durch die EMA im Jahre 2010. Die Preisgestaltung hat z. B. in Großbritannien dazu geführt, dass der bisherige Rahmen (ca. $600 \mathrm{E}$ pro Jahr pro Patient) auf $60.000 £$ angestiegen ist, ähnlich sieht es in Deutschland, neuerdings auch in den USA [22], aus.

\section{Angeborene myasthenische Syndrome (CMS)}

Die angeborenen myasthenischen Syndrome (congenital myasthenic syndrome) sind genetisch determiniert, sämtlich ultra rare diseases (Prävalenz: 1-2/500000 [9]), ihr Spektrum wird zunehmend größer [20]. Es stehen außer der zugelassenen Therapie mit Pyridostigminbromid keine weiteren Medikamente zur Verfügung, die explizit für eine dieser CMS-Erkrankungen zugelassen sind. Pyridostigminbromid ist aber nur für einige wenige CMS sinnvoll. Die Gabe von 3,4-Diaminopyridin (Amifampridin), das bei einzelnen CMS aufgrund der bekannten Pathophysiologie wirksam ist, muss über Antrag bei der Kasse als Off-Label-Use verordnet werden. Andere teilweise nur über die internationale Apotheke beziehbare Substanzen (z. B. Ephedrin $[32,43])$ stellen Arzt und Patienten vor die gleichen Anforderungen wie ein Off-Label-Use. Eine solche Dauertherapie nach differenzierter Untersuchung, z.B. in einem neuropädiatrischen iMZ oder einer geeigneten spezialisierten Muskelambulanz, ist in jedem Fall erforderlich [44].

\section{Pragmatisches Vorgehen beim Einsatz von Substanzen im Off-Label-Use bei Myasthenia gravis, LEMS und CMS}

1. Die Immunsuppressiva Methorexat und Ciclosporin A sind für den Einsatz bei Myasthenia gravis oder LEMS stets ein Off-Label-Use, außer in Fällen mit einer rheumatischen Zweiterkrankung. Da sie in der Rheumatherapie anerkannte
Medikamente mit breitem Einsatz sind, allerdings auch hier z. T. Off-Label, und die Kenntnis und das Verständnis des MDK somit vorhanden sind, entstehen nur ausnahmsweise Probleme bei der Kostenübernahme.

2. Eine intermittierende Therapie mit IVIG als Langzeit-Therapie ist eine seltene Indikation als Add-on-Therapie in Krankheitsphasen, in denen eine stabilisierende Intervention erforderlich ist. Da dies bislang ein Off-Label-Use ist, muss der Einzelfall differenziert dargelegt werden. Mit einer Ablehnung durch den MDK ist leider zurechnen.

3. Der Einsatz von Rituximab (Off-Label-Use) ist der Versuch einer Remissionsinduktion bei therapierefraktärer Myasthenia gravis. Diese bedeutsame Eskalationstherapie im „ultra rare disease“-Bereich ist im Vorfeld einer ambulanten Langzeittherapie unbedingt zu beantragen. Hier ist die Reputation eines spezialisierten Zentrums anzuraten.

4. Die Indikation von Eculizumab als Add-on-Therapie bei therapierefraktärer generalisierter Myasthenia gravis ist auf Patienten mit Acetylcholinrezeptor-Antikörper-Nachweis begrenzt.

5. Die Indikationsfindung für eine Add-on-Therapie mit Eculizumab sollte über ausgewiesene Myasthenie-Zentren erfolgen.

6. 3,4-Diaminopyridin (Amifampridin) kann derzeit bei LEMS weiterhin nach dem Neuen Rezept Formularium (NRF-Ziffer 22.3.) durch Apotheken angefertigt werden. Die ambulante Verordnung des Originalpräparats bedarf einer entsprechenden Bemühung für einen Sonderstatus der Praxis (sog. kostenintensiver Patient).

\section{Interessenkonflikt}

Ordentliches Mitglied der Arzneimittelkommission der deutschen Ärzteschaft (AkdÄ), Mitglied der Expertengruppe Off-Label (Neurologie/Psychiatrie) am BfArM.

\section{Englische Version}

Dieser Beitrag wurde auf Englisch publiziert in Neurology International Open 2018; 2: E118-E123.

\section{Literatur}

[1] Bundesinstitut für Arzneimittel und Medizinprodukte (BfArM). www.bfarm.de

[2] Erhebung des Off-Label-Use in der EU. 2017: www.https://ec.europa. eu/...2017_2_28_final study_report_on_off-label_use.pdf

[3] Janzen RWC, Ludwig WD. Off-Label-Therapie: aktuelle Probleme aus Sicht der Arzneimittelkommission der deutschen Ärzteschaft. Z Rheumatol 2012; 71: 108-118

[4] Clemens T. Zulässigkeit von Arzneiverordnungen und Kostenregresse gegen Ärzte - Off-Label-Use und Unlicensed Use. GesR 07 2011: 397-409

[5] Bundessozialgericht (B 1 KR 1/16 R). 13.12. 2016 
[6] Bundesverfassungsgericht (1 BvR 452/17). 11.04. 2017

[7] Janzen RWC. Off-Label-Use im Behandlungsalltag. Arzneiverordnungen in der Praxis. 43(2016)127-132

[8] European Federation of Neurological Associations (EFNA) board Declaration: Good Off-Label Use Practices (GOLUP). 2017: www.braincouncil.eu/activities/golup

[9] Orphanet.www.orpha.net/national/DE-DE/index/startseite

[10] Boldingh MI, Maniaol AH, Brunborg C et al. Geographical distribution of myasthenia gravis in Northern Europe - Results from a populationbased study from two countries. Neuroepidemiology 2015; 44: 221 231

[11] Breiner A, Widdifield J, Katzberg HD et al. Epidemiology of myasthenia gravis in Ontario, Canada. Neuromuscul Dis 2016; 26: 41-46

[12] Müller-Oerlinghausen B, Schulz M. Abruptes Ende der systematischen Aufbereitung des Altarzneimittelmarkts im Bereich Psychiatrie, Neurologie und Anästhesie. Nervenarzt 1996; 67: 1040-1045

[13] Gemeinsamer Bundesausschuss (G-BA). www.g-ba.de

[14] (DGN). Leitlinien der Deutschen Gesellschaft für Neurologie. 01.04. 2017: www.dgn.org

[15] Andersen JB, Owe JF, Engeland A et al. Total drug treatment and comorbidity in myasthenia gravis: a population-based cohort study. Eur J Neurol 2014; 21: $948-955$

[16] Wöhrle G. Untersuchungen zu Epidemiologie und Verlauf der Myasthenia gravis in Deutschland. Dissertation, TU Darmstadt: 2000

[17] Iorio R, Damato V, Albioni PE et al. Efficacy and safety of rituximab for myasthenia gravis: a systematic reviews and meta-analysis. J Neurol 2015; 262: 1115-1119

[18] Gilhus NE, Verschuur JJ. Myasthenia gravis: subgroup classification and therapeutic strategies. Lancet Neurol 2015; 14: 1023 - 1036

[19] Dalakas MC. Novel future therapeutic options in myasthenia gravis. Autoimmun Rev 2013; 12: 936 - 941

[20] Engel AG, Shen XM, Selcen D et al. Congenital myasthenic syndromes: pathogenesis, diagnosis, and treatment. Lancet Neurol 2015; 14: $420-434$

[21] Vrinten C, Lipka AF, van Zwet EW et al. Ephedrine as add-on therapy for patients with myasthenia gravis: protocol for a series of randomised, placebo-controlled n-of-1 trials. BMJ Open 2015; 5: e007863 doi:10.1136/bmjopen-2015-007863

[22] Burns TM, Smith GA, Allen JA et al. Editorial by concerned physicians: Unintended effect of the Orphan Drug Act on the potential cost of 3,4-diaminopyridine. Muscle Nerve 2016; 53: 165- 168

[23] Howard JF, Barohn RJ, Cutter GR et al. A randomized, double-blind, placebo-controled phase II study of eculizumab in patients with refractory generalized myasthenia gravis. Muscle Nerve 2013; 48 : $76-84$

[24] Howard JF Jr., Freimer M, O'Brien F et al. QMG and MG-ADL correlations: Study of eculizumab treatment of myasthenia gravis. Muscle Nerve 2017; 56: $328-330$

[25] 30.07. 2017: www.ema.eu/docs/en_GB/document_library/summary_of_opinion/000791/WC500229915.pdf
[26] Bungard S, Rohn H, Döbler K. Zertifizierung von Myastheniezentren: Entwicklung und Implementierung eines Zertifizierungsprozesses für Selbsthilfeorganisationen. Z Evid Fortbild Qual Gesundhwes 2011; 105: 49-53

[27] Pohanka M. Acetylcholinesterase inhibitors: a patent review (2008present). Exp Opin Ther Pat 2012; 22: $871-886$

[28] Henze T, Janzen RWC, Schumm F et al. Immuntherapie bei Myasthenia gravis und Lambert-Eaton-Syndrom. Teil 1: Medikamentöse Immunsuppression. Akt Neurol 2010; 37: 505-517

[29] Urban P, Jacobi C, Jander S. Therapiestandards und individualisierte Therapie bei der Myasthenia gravis. Akt Neurol 2018; 45: 253-262

[30] Silvestri NJ, Palace JA. Recognizing refractory myasthenia gravis. Fast Facts. Oxford: Health Press Limited; 2017

[31] Suh J, Goldstein JM, Nowak RJ. Clinical characteristics of refractory myasthenia gravis patients. Yale J Biol Med 2013; 86: 255-260

[32] Sieb JP. Myasthenia gravis: an update for the clinician. Clin Exp Immunol 2014; 175: $408-418$

[33] Alshekhlee A, Miles JD, Katirji B et al. Incidence and mortality rates of myasthenia gravis and myasthenic crisis in US hospitals. Neurology 2009; $72: 1548-1554$

[34] Silvestri NJ, Wolfe GI. Treatment-refractory myasthenia gravis. J Clin Neuromuscul Dis 2014; 15: 167 - 178

[35] Della Marina A, Tripp H, Lutz S et al. Juvenile myasthenia gravis: recommendations for diagnostic approaches and treatment. Neuropediatrics 2014; 45 : $75-83$

[36] Wagner S, Janzen RWC, Mohs C et al. Langzeitbehandlung der therapierefraktären Myasthenia gravis mittels Immunadsorption. Dtsch Med Wochenschr 2008; 133: 2377-2382

[37] Robeson KR, Kumar A, Keung B et al. Durability of the rituximab response in acetylcholine receptor autoantibody-positive myasthenia gravis. JAMA Neurol 2017; 74: $60-66$

[38] Tandan R, Hehir MK, Waheed W et al. Rituximab treatment of myasthenia gravis: a systematic review. Muscle Nerve 2017; 56: 185-196

[39] Hoffmann S, Meisel A. Therapierefraktäre Verläufe und Eskalationsstrategien in der Behandlung der Myasthenia gravis. Akt Neurol 2018; 45: $294-297$

[40] Rodríguez Cruz PM, Palace J, Ramjattan H et al. Salbutamol and ephedrine in the treatment of severe AChR deficiency syndromes. Neurology 2015; 85: $1043-1047$

[41] Fernandes Oliveira E, Nacif AH et al. Sleep disorders in patients with myasthenia gravis: a systematic review. J Phys Ther Sci 2015; 27: 2013-2018

[42] Kohler S, Meisel A. Das Lambert-Eaton-Myasthenie-Syndrom: ein Überblick. Akt Neurol 2018; 45: 298-304

[43] Vrinten C, van der Zwaag AM, Weinreich SS et al. Ephedrine for myasthenia gravis, neonatal myasthenia and the congenital myasthenic syndromes. Cochrane Database Syst Rev 2014; 12: CD010028 doi:10.1002/14651858.CD010028.pub2

[44] Schara U, Della Marina A, Abicht A. Congential myasthenic syndromes: current diagnostic and therapeutic approaches. Neuropediatrics 2012; 43: $184-193$ 